\title{
Addressing Integration Error for Polygonal Finite Elements Through Polynomial Projections: A Patch Test Connection
}

\author{
Cameron Talischi, Glaucio H. Paulino* \\ Department of Civil and Environmental Engineering, University of Illinois at Urbana-Champaign, USA \\ *Corresponding author, paulino@uiuc.edu \\ July 16, 2013
}

\begin{abstract}
Polygonal finite elements generally do not pass the patch test as a result of quadrature error in the evaluation of weak form integrals. In this work, we examine the consequences of lack of polynomial consistency and show that it can lead to a deterioration of convergence of the finite element solutions. We propose a general remedy, inspired by techniques in the recent literature of mimetic finite differences, for restoring consistency and thereby ensuring the satisfaction of the patch test and recovering optimal rates of convergence. The proposed approach, based on polynomial projections of the basis functions, allows for the use of moderate number of integration points and brings the computational cost of polygonal finite elements closer to that of the commonly used linear triangles and bilinear quadrilaterals. Numerical studies of a two-dimensional scalar diffusion problem accompany the theoretical considerations.

Keywords: polygonal and polyhedral meshes, finite elements, patch test, quadrature error, mimetic finite differences
\end{abstract}

\section{Introduction}

Polygonal finite elements, whose development dates back to the seminal work of Wachspress [45], have gained in popularity as evidenced by the growing literature on the topic (see, for example, [37, 36, 34, 20, 43]). Among the attractive features of polygonal elements is the greater flexibility they offer in mesh generation. For example, recently developed algorithms utilize Voronoi diagrams to generate polygonal and polyhedral grids with desired regularity and size distribution for complex geometries [42, 17, 10]. Owing to their high degree of isotropy, these Voronoi meshes have been recently used in dynamic fracture simulations to reduce mesh bias in computed crack patterns [9, 23]. In these analyses, cracks propagate along element boundaries and commonly-used simplicial meshes possess preferential crack path directions [29, 33]. The availability of polygonal finite elements also simplifies mesh adaption procedures such as local refinement (through element-splitting) and coarsening (through aggregation) since hanging nodes are naturally accommodated [39, 32, 8, 4]. In several applications, discretization methods on polygonal and polyhedral 
grids exhibit improved stability and accuracy when compared to their simplicial and cubical counterparts. For example, a low-order finite element scheme defined for large class of polygonal meshes has been shown to be stable for incompressible flow problems [6, 44]. Similarly, polygonal elements can exclude checkerboard layouts and other numerical instabilities that plague the finite element solution of topology optimization problems [40, 41]. In terms of accuracy, mixed polygonal finite elements can be more effective than some commonly-used elements for analysis of incompressible media [44]. Further development of the field can also contribute to the advancement of compatible or structure-preserving methods that require computations on dual grids made up of polygonal and polyhedral cells [21, 16, 19, 22].

The present work deals with the issue of numerical integration for polygonal finite elements, necessary for the evaluation of weak form integrals, and its implications for accuracy of the resulting approximations. Numerical integration for polygonal elements is different from classical triangular and quadrilateral finite elements for two reasons. First, few tailored quadrature schemes are available in the literature owing to the arbitrary geometry of the element domain (see [25, 26] and references therein). In practice, a simple but perhaps sub-optimal procedure is often adopted wherein each polygon is divided into triangular subdomains and the usual quadrature rules are used in each subdomain. Second, all the available quadrature schemes are generally inexact even on regular $n$-gons due to the nonpolynomial nature of the basis functions. One consequence is that the patch test is not passed unless, of course, a very high-order quadrature rule is used to lower the errors to machine precision levels. Such a quadrature scheme may require hundreds of integration points and thus is not feasible [36].

We will show that the error in the satisfaction of the patch test, in so far as it measures a lack of polynomial consistency of the discrete system, places a limit on the convergence of the finite element solutions. More specifically, the solution error cannot be made smaller with mesh refinement beyond a certain level, thus rendering the method non-convergent. A similar issue also plagues meshless methods as they feature non-polynomial functions and remedies for revival of polynomial consistency have been explored for quite some time now [14, 30, 24]. In a recent series of studies, Babuska, Banerjee and co-workers [2, 3, 46] have shown that, under a zero-sum condition or satisfaction of a discrete Green's identity, the order of quadrature rule has to be increased with refinement in order to retain optimal rates of convergence for meshless discretizations.

In this work, we consider an alternative approach that ensures the satisfaction of the patch test and optimal convergence rates with a fixed but sufficiently rich quadrature rule. In practice, the number of integration points for such a scheme is on the order of the number of nodes and therefore the overall computational cost of the method is on par with the linear triangles and quads. As we will see, we do not need to completely eliminate the integration error in the evaluation of the bilinear form. Instead, what is needed is the consistency of the discrete bilinear form when one of its arguments is a piecewise polynomial field. This is accomplished by splitting the local (elemental) forms according to a polynomial projection of its arguments and performing numerical integration only on the "non-polynomial" part. The subsequent restoration of polynomial consistency is sufficient for the satisfaction of the patch test and recovery of optimal convergence rates. 
We remark that the present context is somewhat simpler compared to meshless methods since the the support of basis functions coincides with element domains and their behavior on element interfaces is known. As a result, the proposed remedy is carried out at the element level and directly extended for higher order discretizations.

The proposed approach borrows heavily from the techniques in the mimetic finite difference (MFD) literature (e.g., [12, 7]) and in particular the recently developed variational scheme labeled the Virtual Element Method or VEM [5, 1]. The polynomial projection and the splitting of the bilinear form used in the present work is in fact at the heart of this method. Since an explicit form of trial and test functions is not available (or required) in VEM, the remaining non-polynomial term in the bilinear form is only estimated. What VEM elucidates is a constructive approach for satisfaction of the patch test, which is sufficient for guaranteeing the convergence of conforming Galerkin-type approximations. As we shall see, the linear polygon with the proposed splitting can be in fact viewed as a particular realization of a first-order Virtual element. The same cannot be said for higher order elements, including the quadratic serendipity elements considered here, as the choice of degrees of freedom will, in general, be different. Similarly, access to the basis functions greatly simplifies our treatment of non-constant coefficients. While a thorough comparison between VEM and comparabl $\varrho^{1}$ finite elements in terms of cost and accuracy merits its own study, we emphasize that the goal of the present work is to reduce the burden of numerical integration for polygonal and polyhedral finite elements, which we hope, will also be beneficial for a broader class of problems (e.g. nonlinear problems such as the Navier-Stokes flow [44]), including those for which either an MFD or VEM formulation presently does not exist.

The remainder of the paper is organized as follows: the model diffusion problem and its finite element approximation are discussed in the next section. We consider the relationship between the quadrature errors and the patch test in section 3. Next, in section 4, we explore the consequences of the integration error in the convergence of finite element approximations and discuss sufficient conditions on the discrete bilinear form to recover optimal convergence rates. We present the proposed splitting of the bilinear form as well as its constructions for linear and quadratic elements in section 5. Finally, in section 6, we will address the case of non-constant diffusion tensor. The paper is concluded with some remarks in section 7 and supplementary material on construction of polygonal finite elements and implementation of the proposed approach in the appendix.

We briefly and partially introduce the notation adopted in this paper. We denote by $H^{k}(\Omega)$ the standard Sobolev space consisting of functions whose $k$ th derivative is squareintegrable over the given domain $\Omega$ and write $\|\cdot\|_{k, \Omega}$ and $|\cdot|_{k, \Omega}$ for its norm and semi-norm. We write $L^{2}(\Omega)=H^{0}(\Omega)$ and denote by $H_{g}^{1}(\Omega)$ functions in $H^{1}(\Omega)$ whose trace on $\partial \Omega$ is equal to $g$. Thus $H_{0}^{1}(\Omega)$ consists of functions that vanish on the boundary of $\Omega$. For any subset $E \subseteq \Omega$, we denote by $|E|$ its Lebesgue measure. The space of polynomials of degree $m$ over $E$ is denoted by $\mathcal{P}_{m}(E)$. Finally, an integral evaluated numerically using a

\footnotetext{
${ }^{1} \mathrm{~A}$ remarkable feature of VEM and related MFD formulations is the systematic construction of elements with arbitrary order of polynomial accuracy and continuity on general shapes. Developing comparable finite elements would be a formidable task.
} 
quadrature rule is indicated by $f$, assuming that location of integration points and weights is clear from the context.

\section{Model problem and finite element approximation}

For the sake of concreteness, we focus on a scalar diffusion problem in two dimensions and limit the discussion to first and second-order polygonal finite elements. Let $\Omega \subseteq \mathbb{R}^{2}$ be a bounded open domain with polygonal boundary and consider the steady state diffusion problem given by

$$
\begin{aligned}
-\operatorname{div}(\mathbb{K} \nabla u) & =f & & \text { in } \Omega \\
u & =g & & \text { on } \partial \Omega
\end{aligned}
$$

where the source $f \in L^{2}(\Omega)$ and boundary data $g \in H^{1 / 2}(\partial \Omega)$ are prescribed. For the moment, we assume that $\mathbb{K}$ is a symmetric, positive definite diffusion tensor that is constant over $\Omega$, and postpone the treatment of the general case of variable coefficients to section 6 .

The variational form of the system of equations (1)-(2) consists of finding $u \in H_{g}^{1}(\Omega)$ such that

$$
a(u, v)=\ell(v), \quad \forall v \in H_{0}^{1}(\Omega)
$$

where the bilinear and linear forms are defined, respectively, by

$$
a(u, v)=\int_{\Omega} \nabla u \cdot \mathbb{K} \nabla v \mathrm{~d} \boldsymbol{x}, \quad \ell(v)=\int_{\Omega} f v \mathrm{~d} \boldsymbol{x}
$$

Note that the coercivity of the bilinear form follows from positive-definiteness of $\mathbb{K}$ and the fact that $|\cdot|_{1, \Omega}$ defines a norm on $H_{0}^{1}(\Omega)$. Together with continuity of the linear form, a consequence of the regularity assumption on $f$, one can show that the above system has a unique and stable solution.

\subsection{Finite element spaces}

To define the finite element approximation of (3), we consider a mesh $\mathcal{T}_{h}$ of $\Omega$ consisting of non-overlapping convex polygons, with maximum diameter $h$. An $H^{1}$-conforming finite element space associated with the mesh $\mathcal{T}_{h}$ is given by

$$
\mathcal{V}_{h}=\left\{v_{h} \in C^{0}(\bar{\Omega}):\left.v_{h}\right|_{E} \in \mathcal{V}_{m}(E), \forall E \in \mathcal{T}_{h}\right\}
$$

where $\mathcal{V}_{m}(E)$, is a finite-dimensional space of functions over element $E$ such that

$$
\mathcal{V}_{m}(E) \supseteq \mathcal{P}_{m}(E), \quad \forall E \in \mathcal{T}_{h}
$$

This means that any polynomial of order $m$ can be represented by the functions in $\mathcal{V}_{m}(E)$. We will consider linear and quadratic elements that satisfy (6) with $m=1$ and $m=2$, respectively. For an $n$-gon $E$, the space $\mathcal{V}_{1}(E)$ has dimension $n$ with degrees of freedom associated with the vertices of $E$. Similarly, the space $\mathcal{V}_{2}(E)$ is of dimension $2 n$ with additional degrees of freedom associated with mid-points of each edge. 
For the first-order element, the space $\mathcal{V}_{1}(E)$ can be defined as the span of a set of socalled generalized barycentric coordinates associated with $E$. A number of such coordinates are available in the literature [36], all of which by definition, are linearly complete and nonnegative. The desirable Lagrangian (Kronecker-delta) property and linear variation on $\partial E$ follow directly from these two properties [18].

In the numerical studies presented in this work 2 , we will use Wachspress coordinates which, under certain shape-regularity assumptions, yield optimal interpolation estimates. More specifically, assuming existence of uniform bounds for the aspect ratio, vertex count and interior angles, we have:

$$
\left\|u-\pi_{h} u\right\|_{1, \Omega}=\mathcal{O}(h)
$$

for a sufficiently smooth function $u$ [20]. In the above expression, $\pi_{h} u$ denotes the usual nodal interpolation of $u$ [11].

For the second-order element, we will use the construction of the serendipity-like element presented in [31]. The basis functions are obtained from appropriate linear combinations of pairwise products of generalized barycentric coordinates. The resulting element is constructed to satisfy (6), the Kronecker-delta property, and exhibits quadratic variation on the boundary. If Wachspress coordinates are use for the construction, under the same shape-regularity assumptions as before, the estimate

$$
\left\|u-\pi_{h} u\right\|_{1, \Omega}=\mathcal{O}\left(h^{2}\right)
$$

holds provided that $u$ is sufficiently smooth. Additional details on the construction of $\mathcal{V}_{m}(E)$ and the polygonal basis functions can be found in the appendix. If $E$ is a triangle, the bases reduce to the usual ones and $\mathcal{V}_{m}(E)=\mathcal{P}_{m}(E)$.

\subsection{Approximate problem}

Let $\mathcal{V}_{h, g}=\mathcal{V}_{h} \cap H_{g}^{1}(\Omega)$ and $\mathcal{V}_{h, 0}=\mathcal{V}_{h} \cap H_{0}^{1}(\Omega)$ be the discrete test and trial spaces ${ }^{3}$. We consider a finite element approximation of (3) that consists of finding $u_{h} \in \mathcal{V}_{h, g}$ such that

$$
a_{h}\left(u_{h}, v_{h}\right)=\ell\left(v_{h}\right) \quad \forall v_{h} \in \mathcal{V}_{h, 0}
$$

Here $a_{h}: \mathcal{V}_{h} \times \mathcal{V}_{h} \rightarrow \mathbb{R}$ is a discrete bilinear form defined in terms of symmetric local bilinear forms $a_{h}^{E}: \mathcal{V}_{m}(E) \times \mathcal{V}_{m}(E) \rightarrow \mathbb{R}$ as follows

$$
a_{h}(u, v)=\sum_{E \in \mathcal{T}_{h}} a_{h}^{E}(u, v)
$$

These local bilinear forms corresponds to the element stiffness matrices and the summation is related to the assembly process in practice. For instance, when a quadrature rule is used

\footnotetext{
${ }^{2}$ The main results, however, apply to finite elements derived from other barycentric coordinates (e.g. Mean Value, Sibson, Laplace, maximum entropy coordinates).

${ }^{3}$ We are tacitly assuming that $g$ and $\mathcal{T}_{h}$ are defined such that the boundary data can be represented by the trace of functions in $\mathcal{V}_{h}$ and so $\mathcal{V}_{h, g}$ is non-trivial. In general, $g$ must be replaced by its nodal interpolation $g_{h}$ and the test space is set to $\mathcal{V}_{h} \cap H_{g_{h}}^{1}(\Omega)$. However, we will ignore this approximation.
} 
to compute the stiffness matrix, we have

$$
a_{h}^{E}(u, v)=f_{E} \nabla u \cdot \mathbb{K} \nabla v \mathrm{~d} \boldsymbol{x}
$$

If the quadrature in (11) is exact, $a_{h}(u, v)=a(u, v)$, and we recover the classical Galerkin approximation. We will consider alternative constructions of the local bilinear form $a_{h}^{E}$ in section 5. Note that we are assuming in (9) that the exact linear form $\ell$ is available. In practice, numerical integration is usually used to compute this integral, which amounts to replacing $\ell(v)$ by

$$
\ell_{h}(v)=\sum_{E \in \mathcal{T}_{h}} f_{E} f v \mathrm{~d} \boldsymbol{x}
$$

However, since the main difficulty with numerical integration lies in the resulting lack of consistency in the bilinear form, we will ignore this approximation to keep the theoretical discussion simple. In the motivating examples presented in the next two sections, $f \equiv 0$ and numerical integration 12 is in fact exact. Nevertheless comments will be made throughout regarding the effect of this approximation.

In general, we expect that $a_{h}$ inherits the continuity and coercivity properties of $a$. These conditions will be satisfied, for example, if $a_{h}^{E}$ scales as $a^{E}$, that is $4^{4}$

$$
c_{1} a^{E}(v, v) \leq a_{h}^{E}(v, v) \leq c_{2} a^{E}(v, v) \quad \forall v \in \mathcal{V}_{m}(E), \forall E \in \mathcal{T}_{h}
$$

for some positive constants $c_{1}$ and $c_{2}$, independent of $h$ and $E$ [5]. Together with continuity of $\ell$, we can show that (9) admits a unique solution $u_{h}$. Additional consistency requirements on $a_{h}$ are naturally needed to ensure convergence of $u_{h}$ to $u$. The well-known and celebrated patch test provides a means to assess the consistency of the approximation.

\section{Quadrature error and the patch test}

The engineering patch test is performed by applying boundary conditions $g=\left.p\right|_{\partial \Omega}$, with $p \in \mathcal{P}_{m}(\Omega)$, to a patch of finite elements. This corresponds to the problem where the exact solution $u=p$. In this section, we consider the approximate bilinear form defined by numerical integration, cf. (11).

Since $\mathcal{V}_{h} \supseteq \mathcal{P}_{m}(\Omega)$, then $p \in \mathcal{V}_{h, g}$ and we will have $u_{h}=p$ if the quadrature rule in (11) is exact. It can be readily shown that the patch test is also passed if

$$
a_{h}^{E}(p, v)=a^{E}(p, v), \quad \forall v \in \mathcal{V}_{m}(E), \forall E \in \mathcal{T}_{h}
$$

To see this, note that (14) implies

$$
a_{h}\left(p, v_{h}\right)=\sum_{E \in \mathcal{T}_{h}} a_{h}^{E}\left(p, v_{h}\right)=\sum_{E \in \mathcal{T}_{h}} a^{E}\left(p, v_{h}\right)=a\left(p, v_{h}\right)=\ell\left(v_{h}\right)
$$

\footnotetext{
${ }^{4}$ Here $a^{E}$ denotes the restriction of $a$ to element $E$, i.e., $a^{E}(u, v)=\int_{E} \nabla u \cdot \mathbb{K} \nabla v \mathrm{~d} \boldsymbol{x}$.
} 

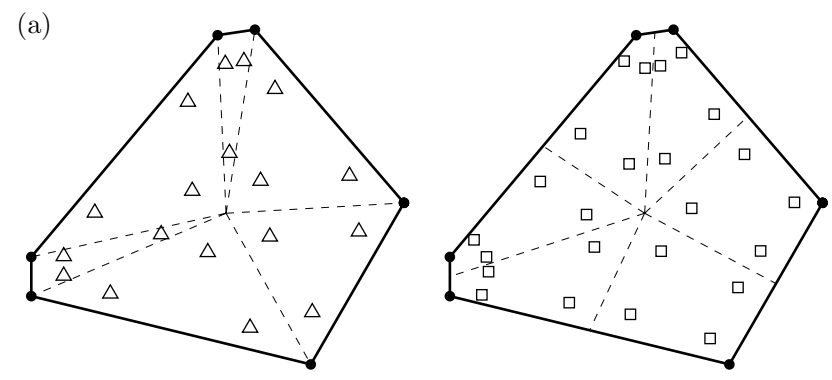

(b)

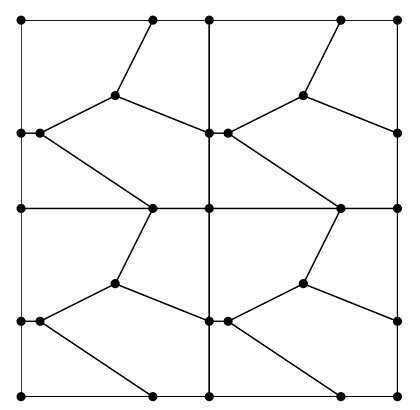

Figure 1: (a) Distribution of integration points for second-order triangulation (left) and quadrangulation (right) integration schemes on a sample hexagon (b) 2nd-level mesh used for the patch test and convergence studies. It consists of $2 \times 2$ patches of two quadrilaterals and two pentagons.

for each $v_{h} \in \mathcal{V}_{h, 0}$, and so $u_{h}=p$ is the unique solution to the discrete problem. Essentially, (14) is a polynomial consistency condition requiring the local bilinear forms to be exact when one of the arguments is a polynomial function.

For $m=1$ and the first order patch test, the above condition can be further simplified. If $\varphi_{1}, \ldots, \varphi_{n}$ denote the basis for $\mathcal{V}_{1}(E)$, for (14) to hold for an arbitrary linear function $p$, we must have

$$
f_{E} \nabla \varphi_{i} \mathrm{~d} \boldsymbol{x}=\int_{E} \nabla \varphi_{i} \mathrm{~d} \boldsymbol{x}, \quad i=1, \ldots, n
$$

Therefore, a sufficient condition for passing the first-order patch test is that the quadrature rule integrates the gradient of the basis functions exactly. This fact that has been noted and used in literature of meshless methods (see, for example, [24]).

Few remarks regarding this observation are in order. First, the classical isoparametric bilinear quadrilateral with $2 \times 2$ Gauss quadrature passes the patch test even though there is error in the integration of discrete bilinear form (i.e., the stiffness matrix) when the elements are angularly distorted. The patch test is passed precisely because (16) holds in such a case, a fact seldom discussed in finite element textbooks. More specifically, if $E$ is the image of the reference element $\hat{E}=[-1,1]^{2}$ under the bilinear map $\boldsymbol{F}$, then the basis functions are defined through the relation $\varphi_{i}=\hat{\varphi}_{i} \circ \boldsymbol{F}^{-1}$ with $\hat{\varphi}_{i}$ the bilinear function on $\hat{E}$ associated with $i$ th vertex. The relation

$$
\int_{E} \nabla \varphi_{i} \mathrm{~d} \boldsymbol{x}=\int_{\hat{E}}(D \boldsymbol{F})^{-1} \hat{\nabla} \hat{\varphi}_{i} \operatorname{det}(D \boldsymbol{F}) \mathrm{d} \hat{\boldsymbol{x}}=\int_{\hat{E}} \operatorname{adj}(D \boldsymbol{F}) \hat{\nabla} \hat{\varphi}_{i} \mathrm{~d} \hat{\boldsymbol{x}}
$$

indicates the integrand of the right-hand side is bilinear function of $\hat{\boldsymbol{x}}$ and so $2 \times 2$ Gauss quadrature on $\hat{E}$ is exact. In the above expression, $D \boldsymbol{F}$ denotes the Jacobian matrix for $\boldsymbol{F}$ and $\operatorname{adj}(D \boldsymbol{F})$ is the transpose of the cofactor of $D \boldsymbol{F}$. Note, however, that the bilinear form (11) will not be exact with this quadrature if $\operatorname{det}(D \boldsymbol{F})$ is not constant.

For a general polygon, including distorted quadrilaterals, the Wachspress basis consisting of rational function $5^{5}$, the relation (16) will not hold with the available quadrature

\footnotetext{
${ }^{5}$ The basis functions for the iso-parametric quads are also rational in the physical coordinates but
} 


\begin{tabular}{|c|c|c|c|c|c|c|}
\hline Quadrature order & 1 & 2 & 4 & 8 & 16 & 32 \\
\hline \hline Triangulation & $6.193 \mathrm{E}-02$ & $1.362 \mathrm{E}-02$ & $7.165 \mathrm{E}-04$ & $2.558 \mathrm{E}-06$ & $4.619 \mathrm{E}-11$ & $1.223 \mathrm{E}-15$ \\
\hline Quadrangulation & $1.766 \mathrm{E}-02$ & $1.074 \mathrm{E}-03$ & $5.945 \mathrm{E}-05$ & $2.206 \mathrm{E}-07$ & $3.437 \mathrm{E}-12$ & $1.860 \mathrm{E}-15$ \\
\hline
\end{tabular}

Table 1: Quadrature error for integration of basis function gradients using different schemes

schemes since they are constructed for integration of polynomials. However, (16) suggests that a quadrature rule that does a better job of the integrating the gradients of the basis functions would have a smaller error in the patch test. As mentioned in the introduction, a simple quadrature scheme for polygonal domains is obtained by triangulation. We will consider an alternative "quadrangulation" procedure, which as shown in Figure 1(a), consists of splitting the $n$-gon into $n$ quadrilaterals by connecting the centroid of the polygon to the midpoint of each edge and using Gauss quadrature (after a bilinear mapping) in each quad. It is evident from the figure that this approach leads to a denser distribution of integrations points along the edges of the element where the basis function gradients are large. By contrast, the triangulation approach has a denser distribution in the interior of the element.

In Table 1, we compare the error in the integration of basis function gradients according to

$$
\max _{i}\left|\int_{E} \nabla \varphi_{i} \mathrm{~d} \boldsymbol{x}-f_{E} \nabla \varphi_{i} \mathrm{~d} \boldsymbol{x}\right|
$$

for the polygon with geometry shown in Figure 1(a). We first note that the error is finite for all the quadrature orders considered and many integration points are needed to drive the error close to machine precision level. Also, the "quadrangulation" scheme leads to smaller errors compared to the triangulation approach, in agreement with the discussion above. We next test to see if the patch test errors follow the same trend.

We perform the first-order patch test on the unit square $\Omega=] 0,1\left[^{2}\right.$ with $u(\boldsymbol{x})=$ $2 x_{1}-x_{2}+4$ and, $\mathbb{K}$ taken to be the identity matrix, on a sequence of polygonal meshes. As shown in Figure 1(b), the $k$ th level mesh consists of $2^{k-1} \times 2^{k-1}$ patches of two quadrilaterals and two pentagons. Observe the source function associated with $u$ vanishes, i.e., $f \equiv$ $-\nabla \cdot(\mathbb{K} \nabla u)=0$. The reported errors in Figure $2\left(\right.$ a) are with respect to the $L^{2}$-norm and $H^{1}$-seminorm, given by

$$
\epsilon_{0}(h)=\frac{\left\|u-u_{h}\right\|_{0, \Omega}}{\|u\|_{0, \Omega}}, \quad \epsilon_{1}(h)=\frac{\left|u-u_{h}\right|_{1, \Omega}}{|u|_{1, \Omega}}
$$

respectively. Note that $\epsilon_{1}(h)$ is the same as the error in the energy norm for this problem. The results show smaller errors with the quadrangulation scheme, in agreement with the discussion above and results of Table 1 . We also note that, while the $L^{2}$-error evidently goes to zero with $h$, the error in the energy norm does not vanish, indicating that the patch test is not passed even in a "weak" sense. Though not presented, we have observed the same behavior for higher order quadrature rules.

they are images of a polynomial functions under a polynomial transformation. In fact, the gradient of the Wachspress basis functions for a general quadrilateral will not be integrated exactly with Gauss quadrature. 

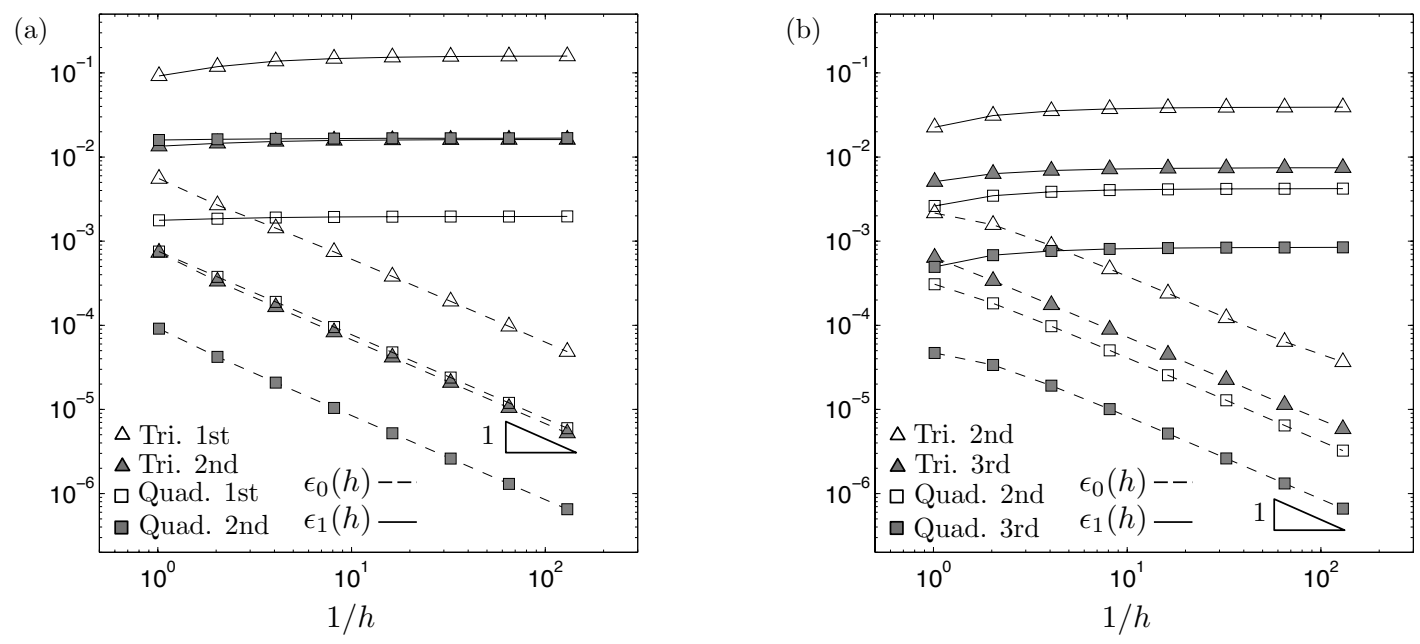

Figure 2: Results of (a) the linear patch test (b) the quadratic patch test using indicated quadrature schemes (the legend shows the type of subdivision and order of quadrature in each subdomain)

The above study can be extended to quadratic elements for which condition (14) reduces to a set of conditions on the integration of basis function gradients, similar to (16), and their moments. We can verify that the errors in the patch test also correlate with the accuracy of the quadrature scheme for evaluation of the gradients.

We will only present the results for a quadratic patch on the same sequence of meshes as in the previous example. The exact solution is $u(\boldsymbol{x})=x_{1}^{2}-3 x_{1} x_{2}-x_{2}^{2}+5 x_{1}$ with diffusion tensor taken as the identity and $f \equiv 0$. Second and third order quadrature rules are used for the triangular and quadrilateral subdomains. We observe, from the results shown in Figure 2(b), that the quadrangulation scheme again leads to smaller errors that the triangulation approach. Also, the energy norm errors do not decrease with mesh refinement, while the $L^{2}$-errors decrease with a linear rate in the range of mesh sizes considered.

\section{Effects of quadrature error on convergence}

The persistent errors in the patch test under mesh refinement, revealed in the numerical study of previous section, also indicate the finite element approximations obtained from (11) using a fixed quadrature are not convergent. Simply put, if solutions do not converge when the exact solution is a polynomial, the method cannot be deemed convergent in general. This is an alarming observation and, to the best of our knowledge, not discussed explicitly in the literature on polygonal finite elements.

To further explore the influence of integration error on the convergence of the approximations, we consider the problem with exact solution

$$
u(\boldsymbol{x})=\sin \left(x_{1}\right) \exp \left(x_{2}\right)
$$

$\mathbb{K}$ taken as the identity matrix, and $f(\boldsymbol{x}) \equiv 0$ on the unit square. The boundary data $g$ is specified in accordance with (20). The results for the same regular sequence of meshes 

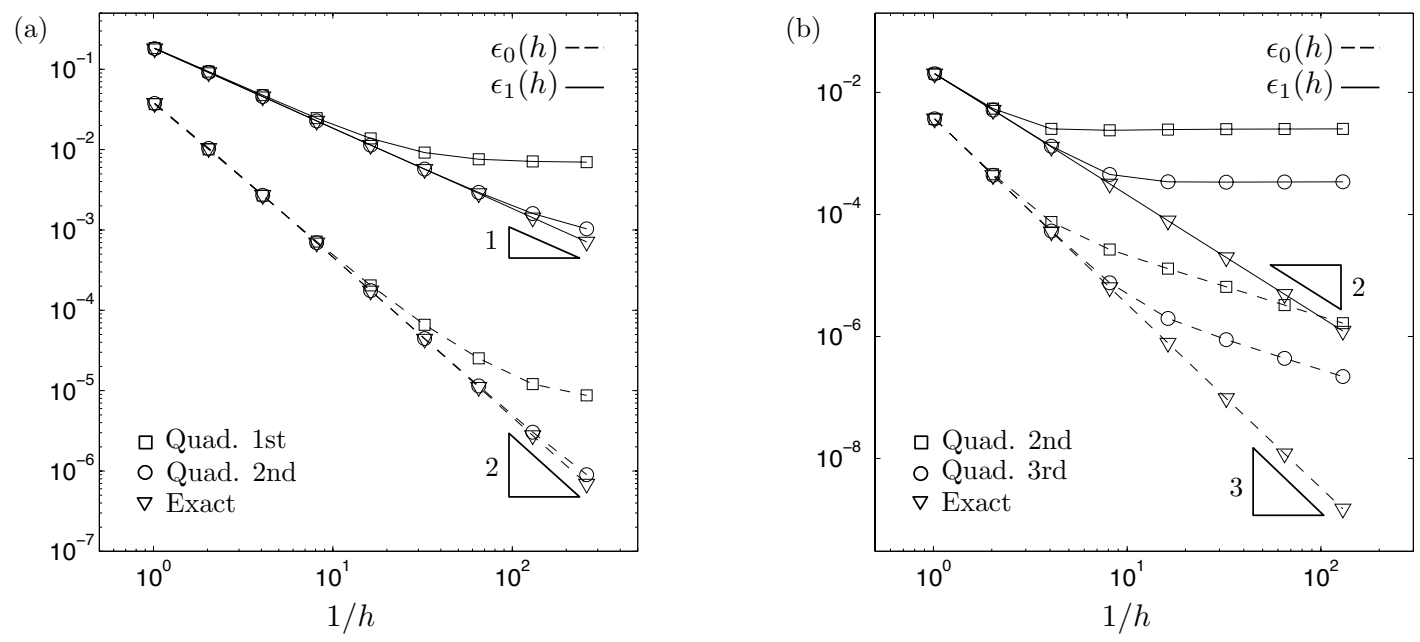

Figure 3: Results of the convergence study with (a) linear elements (b) quadratic elements. In both cases, the quadrangulation scheme with indicated integration order is used

using the quadrangulation scheme is summarized in Figure 3. While we see optimal rates of convergence with "exact" integration ${ }^{6}$ quadrature error leads to a severe degradation of convergence in the $L^{2}$-norm and a lack of convergence of in the energy norm. The onset of this poor behavior in fact correlates with the observed error in the patch test. These results confirm that the patch test error places a limit on the accuracy that can be achieved by the finite element solution. We remark that this convergence behavior is reminiscent of error trends for meshless discretizations presented in reference [46].

We proceed next to discuss a variation of Strang's first lemma (cf. [15]), that, on the one hand, is in agreement with the above observation, and, on the other, proves that the satisfaction of the local consistency condition (14), along with (13), is sufficient to ensure optimal convergence.

As before, let $\pi_{h}$ be the nodal interpolant on $\mathcal{V}_{h}$. We define $\tau_{h} u$ to be a piecewise polynomial field on $\mathcal{T}_{h}$ that best approximates $u$ with respect to the discrete semi-norm

$$
|\cdot|_{h}^{2}:=\sum_{E \in \mathcal{T}_{h}}|\cdot|_{1, E}^{2}
$$

This means that for each $E \in \mathcal{T}_{h}$, the restriction of $\tau_{h} u$ to $E$ belongs to $\mathcal{P}_{m}(E)$ and

$$
\left.\tau_{h} u\right|_{E}=\underset{p \in \mathcal{P}_{m}(E)}{\operatorname{argmin}}|u-p|_{1, E}
$$

Viewed another way, $\left.\left(\nabla \tau_{h} u\right)\right|_{E}$ is the $L^{2}$-projection of $\nabla u$ onto $\left[\mathcal{P}_{m-1}(E)\right]^{2}$. Note that $\tau_{h} u$ is not necessarily continuous across element boundaries and need not belong to $H^{1}(\Omega)$. For sufficiently smooth $u$ and under the shape-regularity assumptions on the elements in $\mathcal{T}_{h}$, one can show that $\left|u-\tau_{h} u\right|_{h}=\mathcal{O}\left(h^{m}\right)[11$.

\footnotetext{
${ }^{6}$ These results are obtained using very high order quadrature such that error in the calculation of the bilinear form is close to machine precision levels.
} 
Provided that the discrete bilinear form satisfies (13), we have the following a priori estimate for the approximation error:

$$
\left|u-u_{h}\right|_{1, \Omega} \leq C\left(\left|u-\pi_{h} u\right|_{1, \Omega}+\left|u-\tau_{h} u\right|_{h}+\sup _{v_{h} \in \mathcal{V}_{h, 0}} \sum_{E \in \mathcal{T}_{h}} \frac{\left|a_{h}^{E}\left(\tau_{h} u, v_{h}\right)-a^{E}\left(\tau_{h} u, v_{h}\right)\right|}{\left|v_{h}\right|_{1, \Omega}}\right)
$$

where $C$ is a constant independent of $h$. The ingredients for its proof can be found in the proof of Theorem 3.1 in [5] and will not be repeated here. In fact, (23) is at the core of convergence of VEM.

The first two terms are interpolation errors of order $\mathcal{O}\left(h^{m}\right)$. Noting that $\left.\tau_{h} u\right|_{E} \in \mathcal{P}_{m}(E)$, the remaining term, involving the approximation of the bilinear form, is closely related to the errors in respecting the consistency condition (14). If the discrete bilinear form satisfies (14), this term vanishes and one obtains an overall error bound of $\left|u-u_{h}\right|_{1, \Omega}=\mathcal{O}\left(h^{m}\right)$, which is optimal. One retains optimal convergence rates even if consistency error in the approximation of bilinear form is $\mathcal{O}\left(h^{m}\right)$, that is, if

$$
\left|a_{h}^{E}(p, v)-a^{E}(p, v)\right| \leq C h^{m}|v|_{1, E} \quad \forall p \in \mathcal{P}_{m}(E), \forall v \in \mathcal{V}_{m}(E), \forall E \in \mathcal{T}_{h}
$$

with the constant $C$ independent of $h$ and $E$.

We note that the estimate (23) sheds light on convergence behavior observed in the numerical examples presented in the beginning of this section. For coarser meshes, the interpolation errors, represented by the first two terms in $(23)$, dominate while for sufficiently small $h$, the consistency error in approximation of the bilinear form controls the overall error. Thus, the degradation in convergence is "delayed" if the consistency error is lowered. However, the finite consistency error that accompanies any fixed inexact quadrature rule will ultimately dominate.

Let us also remark that if the linear form $\ell(v)$ is approximated through quadrature by (12), an additional term of the form

$$
\sup _{v_{h} \in \mathcal{V}_{h, 0}} \frac{\left|\ell_{h}\left(v_{h}\right)-\ell(v)\right|}{\left|v_{h}\right|_{1, \Omega}}
$$

will appear in the estimate (23). However, provided that the quadrature integrates constant functions exactly on each element of the mesh and $f$ is sufficiently smooth, the error introduced is $\mathcal{O}\left(h^{2}\right)$ (cf. (40) in section 5.2) and thus will not affect the rate of convergence of both linear and quadratic discretizations.

\section{Restoring polynomial consistency}

We now discuss an approach to ensure polynomial consistency even when using a fixed (but inexact) quadrature rule. The proposed approach uses a particular representation of the bilinear form $a^{E}$ that is at central to VEM [5] and effectively nodal MFD [12, 7].

Keeping (6) in mind, we consider a projection operator $\Pi_{m}^{E}: \mathcal{V}_{m}(E) \rightarrow \mathcal{P}_{m}(E)$ for each element $E \in \mathcal{T}_{h}$ such that

$$
\left\{\begin{array}{l}
a^{E}\left(p, \Pi_{m}^{E} v\right)=a^{E}(p, v) \quad \forall p \in \mathcal{P}_{m}(E) \\
\Pi_{m}^{E} p=p
\end{array}\right.
$$


Thus $\Pi_{m}^{E} v$ can be thought of as a polynomial approximation to $v$, as seen by the local bilinear form, minimizing $a^{E}(v-p, v-p)$ in $\mathcal{P}_{m}(E)$. While the above definition is applicable to other elliptic problems such as elasticity, in the present context with $\mathbb{K}$ a constant tensor, $\nabla \Pi_{m}^{E} v$ is the least-squares approximation to $\nabla v$ in $\left[\mathcal{P}_{m-1}(E)\right]^{2}$ :

$$
\begin{aligned}
\nabla \Pi_{m}^{E} v & =\underset{\boldsymbol{q} \in\left[\mathcal{P}_{m-1}(E)\right]^{2}}{\operatorname{argmin}} \int_{E}(\boldsymbol{q}-\nabla v) \cdot \mathbb{K}(\boldsymbol{q}-\nabla v) \mathrm{d} \boldsymbol{x} \\
& =\underset{\boldsymbol{q} \in\left[\mathcal{P}_{m-1}(E)\right]^{2}}{\operatorname{argmin}} \int_{E}|\boldsymbol{q}-\nabla v|^{2} \mathrm{~d} \boldsymbol{x}
\end{aligned}
$$

Also, we observe that for a triangular element $E, \Pi_{m}^{E}$ reduces to the identity map since $\mathcal{V}_{m}(E)=\mathcal{P}_{m}(E)$.

We can use (26), along with the symmetry and linearity of the bilinear form, to show that for $u, v \in \mathcal{V}_{m}(E)$, that $a^{E}(u, v)$ can be split up as,

$$
\begin{aligned}
a^{E}(u, v) & =a^{E}\left(\Pi_{m}^{E} u, v\right)+a^{E}\left(u-\Pi_{m}^{E} u, v\right) \\
& =a^{E}\left(\Pi_{m}^{E} u, v\right)+a^{E}\left(u-\Pi_{m}^{E} u, v\right)+a^{E}\left(u-\Pi_{m}^{E} u, \Pi_{m}^{E} v\right) \\
& =a^{E}\left(\Pi_{m}^{E} u, v\right)+a^{E}\left(u-\Pi_{m}^{E} u, v-\Pi_{m}^{E} v\right) \\
& =a^{E}\left(\Pi_{m}^{E} u, \Pi_{m}^{E} v\right)+a^{E}\left(u-\Pi_{m}^{E} u, v-\Pi_{m}^{E} v\right)
\end{aligned}
$$

Observe that the arguments of the first term are polynomial functions.

Inspired by this identity, we define a discrete bilinear form where numerical integration is used to evaluate the second "non-polynomial" term. That is, we set

$$
a_{h}^{E}(u, v):=a^{E}\left(\Pi_{m}^{E} u, \Pi_{m}^{E} v\right)+f_{E} \nabla\left(u-\Pi_{m}^{E} u\right) \cdot \mathbb{K} \nabla\left(v-\Pi_{m}^{E} v\right) \mathrm{d} \boldsymbol{x}
$$

First, we note that once an explicit expression for $\Pi_{m}^{E}$ is derived, the first term can be evaluated exactly because its arguments are polynomials. Second, this choice of $a_{h}^{E}$ respects the consistency condition (14) since for $u=p \in \mathcal{P}_{m}(E)$, we have $\Pi_{m}^{E} p=p$ and so

$$
a_{h}^{E}(p, v)=a^{E}\left(p, \Pi_{m}^{E} v\right)+f_{E} \nabla(p-p) \cdot \mathbb{K} \nabla\left(v-\Pi_{m}^{E} v\right) \mathrm{d} \boldsymbol{x}=a^{E}\left(p, \Pi_{m}^{E} v\right)=a^{E}(p, v)
$$

The other requirement on the bilinear form, namely condition (13), will be satisfied if a sufficiently rich quadrature scheme is used for the second term in $a_{h}^{E}$. For example, our numerical studies confirm that even the lowest order quadrature schemes (triangulation and quadrangulation) with $n$ integration points are sufficient for this purpose for the linear elements. In the remainder of this section, we will discuss how the projection map and the discrete bilinear form can be computed.

\subsection{Linear elements}

Another key observation made in [5] is that the right-hand-side of (26) can be computed exactly given our knowledge of behavior of functions in $\mathcal{V}_{1}(E)$. Indeed, a simple use of 
integration by parts shows that for $p \in \mathcal{P}_{1}(E)$ and $v \in \mathcal{V}_{1}(E)$

$$
\begin{aligned}
a^{E}(p, v) & =\int_{E} \nabla v \cdot \mathbb{K} \nabla p \mathrm{~d} \boldsymbol{x} \\
& =-\int_{E} v \operatorname{div}(\mathbb{K} \nabla p) \mathrm{d} \boldsymbol{x}+\int_{\partial E} v \mathbb{K} \nabla p \cdot \boldsymbol{n} \mathrm{d} s \\
& =\int_{\partial E} v \mathbb{K} \nabla p \cdot \boldsymbol{n} \mathrm{d} s
\end{aligned}
$$

where we have used $\operatorname{div}(\mathbb{K} \nabla p)=0$ for the second equality. Observe that the last integral can be computed exactly since $v$ varies linearly on the boundary of $E$.

To get an explicit expression for $\Pi_{1}^{E}$, let us set $\boldsymbol{q}=\mathbb{K} \nabla p$ in 31 to get

$$
\int_{E} \nabla \Pi_{1}^{E} v \cdot \boldsymbol{q} \mathrm{d} \mathbf{x}=a^{E}\left(p, \Pi_{1}^{E} v\right)=a^{E}(p, v)=\int_{\partial E} v \boldsymbol{q} \cdot \boldsymbol{n} \mathrm{d} s
$$

Because (26) must hold for all $p \in \mathcal{P}_{1}(E)$, we can choose $p$ to recover any arbitrary constant vector $\boldsymbol{q} \in\left[\mathcal{P}_{0}(E)\right]^{2}$ and therefore, 32 implies,

$$
\int_{E} \nabla \Pi_{1}^{E} v \mathrm{~d} \mathbf{x}=\int_{\partial E} v \boldsymbol{n} \mathrm{d} s
$$

Again observe that the value of the right-hand-side integral depends only on the nodal values of $v$ and the geometry of $E$. Moreover, as $\nabla \Pi_{1}^{E} v$ is a constant vector over $E$, it can be pulled outside of the integral

$$
\nabla \Pi_{1}^{E} v=\frac{1}{|E|} \int_{\partial E} v \boldsymbol{n} \mathrm{d} s
$$

This relation could also be seen directly from 27 since the best constant approximation to $\nabla v$ over $E$ is $|E|^{-1} \int_{E} \nabla v \mathrm{~d} \boldsymbol{x}=|E|^{-1} \int_{\partial E} v \boldsymbol{n} \mathrm{d} s$.

To complete the construction of $\Pi_{1}^{E}$, we assign an appropriate constant in order to respect the condition $\Pi_{1}^{E} p=p$. We can choose the constant, for example, for equating $\int_{\partial E} v \mathrm{~d} s=\int_{\partial E} \Pi_{1}^{E} v \mathrm{~d} s$ or the nodal averages. With the latter choice, we have

$$
\left(\Pi_{1}^{E} v\right)(\boldsymbol{x}):=\bar{v}+\left(\frac{1}{|E|} \int_{\partial E} v \boldsymbol{n} \mathrm{d} s\right) \cdot(\boldsymbol{x}-\overline{\boldsymbol{x}})
$$

where the constant $\bar{v}$ is the mean of the nodal values of $v$ and $\overline{\boldsymbol{x}}$ is the center of $E$ (mean of the location of vertices of $E$ ). Clearly the gradient of (35) satisfies (34), and for $p(\boldsymbol{x})=\alpha+\boldsymbol{\beta} \cdot \boldsymbol{x}$

$$
\begin{aligned}
\left(\Pi_{1}^{E} p\right)(\boldsymbol{x}) & =\bar{p}+\left(\frac{1}{|E|} \int_{E} \nabla p \mathrm{~d} \boldsymbol{x}\right) \cdot(\boldsymbol{x}-\overline{\boldsymbol{x}}) \\
& =(\alpha+\boldsymbol{\beta} \cdot \overline{\boldsymbol{x}})+\boldsymbol{\beta} \cdot(\boldsymbol{x}-\overline{\boldsymbol{x}}) \\
& =p(\boldsymbol{x})
\end{aligned}
$$

verifying that projection map fixes $\mathcal{P}_{1}(E)$. 
As a consequence of the form of $\Pi_{1}^{E}$, and the choice of degrees of freedom for the linear element, the first term in discrete bilinear form (29) does not depend on the form of the basis functions inside the element and is only a function of the geometry of $E$ and diffusion tensor $\mathbb{K}$. This means that elements based on other barycentric coordinates, as well as the first order VEM formulation, all lead to the same "consistency" term.

We also note that if the quadrature scheme satisfies the gradient condition (16), then the discrete bilinear form defined by quadrature (i.e., equation (11) in section 3), is identical to the discrete bilinear form 29 . Indeed,

$$
\begin{aligned}
a_{h}^{E}(u, v) & =a^{E}\left(\Pi_{1}^{E} u, \Pi_{1}^{E} v\right)+f_{E} \nabla\left(u-\Pi_{1}^{E} u\right) \cdot \mathbb{K} \nabla\left(v-\Pi_{1}^{E} v\right) \mathrm{d} \boldsymbol{x} \\
& =2 a^{E}\left(\Pi_{1}^{E} u, \Pi_{1}^{E} v\right)-f_{E} \nabla u \cdot \mathbb{K} \nabla \Pi_{1}^{E} v \mathrm{~d} \boldsymbol{x}-f_{E} \nabla \Pi_{1}^{E} u \cdot \mathbb{K} \nabla v \mathrm{~d} \boldsymbol{x}+f_{E} \nabla u \cdot \mathbb{K} \nabla v \mathrm{~d} \boldsymbol{x} \\
& =2 a^{E}\left(\Pi_{1}^{E} u, \Pi_{1}^{E} v\right)-a^{E}\left(u, \Pi_{1}^{E} v\right)-a^{E}\left(\Pi_{1}^{E} u, v\right)+f_{E} \nabla u \cdot \mathbb{K} \nabla v \mathrm{~d} \boldsymbol{x} \\
& =f_{E} \nabla u \cdot \mathbb{K} \nabla v \mathrm{~d} \boldsymbol{x}
\end{aligned}
$$

This implies that, for isoparameteric bilinear quads, applying quadrature to either representation of the local bilinear form yields the same result.

\subsection{Quadratic elements}

For the serendipity element considered here, $a^{E}(p, v)$ with $p \in \mathcal{P}_{2}(E)$ cannot be reduced to an integral on the boundary of the element. Therefore, numerical quadrature will be needed for the evaluation of an area integral. This can be seen from (31) and the fact that for $p \in \mathcal{P}_{2}(E)$, the quantity $\operatorname{div}(\mathbb{K} \nabla p)$ does not necessarily vanish. However, the quadrature error for computing this term is $\mathcal{O}\left(h^{2}\right)$ since the integrand contains the basis functions and not their gradients. This error is sufficient for ensuring the consistency condition (24) and subsequently maintaining optimal convergence rates.

In this case, we define the discrete bilinear to be again given by 29 , but change the definition of the projection map to

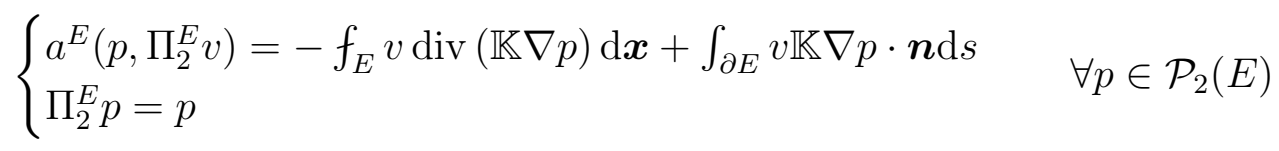

which is a slight deviation from 26) with a revisited right-hand side for the first expression.

As before, the boundary integral in (38) can be computed exactly since the integrand is a polynomial. For the two conditions to be consistent, that is, for the first equality to hold when $v=q \in \mathcal{P}_{2}(E)$, we must require that the quadrature rule is exact for second order polynomials, that is,

$$
f_{E} q \mathrm{~d} \boldsymbol{x}=\int_{E} q \mathrm{~d} \boldsymbol{x}, \quad \forall q \in \mathcal{P}_{2}(E)
$$

This, in particular, indicates that the first order triangulation and quadrangulation schemes consisting of $n$-points will not be sufficient for the quadratic elements and a second-order 
accurate quadrature must be used in the subdomains. These rules have proven in our numerical studies to also be sufficient for ensuring that condition 13 is met. A possible alternative is to use the quadrature rules in [25] which are constructed for exact integration of polynomials on polygonal domains. Compared to subdivision schemes considered here, they require fewer evaluation points to achieve quadratic precision.

To verify the satisfaction of the weaker consistency condition $(24)$, let $p \in \mathcal{P}_{2}(E)$ and set $c \equiv \operatorname{div}(\mathbb{K} \nabla p)$. Then, for $v \in \mathcal{V}_{2}(E)$, we have

$$
\begin{aligned}
\left|a_{h}^{E}(p, v)-a^{E}(p, v)\right| & =\left|a^{E}\left(p, \Pi_{2}^{E} v\right)-a^{E}(p, v)\right| \\
& =\left|\int_{E} v \operatorname{div}(\mathbb{K} \nabla p) \mathrm{d} \boldsymbol{x}-f_{E} v \operatorname{div}(\mathbb{K} \nabla p) \mathrm{d} \boldsymbol{x}\right| \\
& =|c|\left|\int_{E} v \mathrm{~d} \boldsymbol{x}-f_{E} v \mathrm{~d} \boldsymbol{x}\right|
\end{aligned}
$$

The last expression is bounded by $C h^{2}|v|_{1,2, E}$ provided that the quadrature integrates constant functions exactly (see Exercise 4.1.4 of [15]).

The fact that the stronger consistency condition (14) is not satisfied for the quadratic element suggests that the patch test will only be passed asymptotically with mesh refinement. However, we can directly show that the patch test will be passed exactly on any mesh if the approximate linear form $\ell_{h}$ defined by $(12)$ is used with the same quadrature rule as that of the discrete bilinear form. As mentioned before, this is usually the case in practice. Indeed for $p \in \mathcal{P}_{2}(\Omega)$ and $v_{h} \in \mathcal{V}_{h, 0}$, we have

$$
\begin{aligned}
a_{h}\left(p, v_{h}\right) & =\sum_{E \in \mathcal{T}_{h}} a_{h}^{E}\left(p, v_{h}\right) \\
& =\sum_{E \in \mathcal{T}_{h}} a^{E}\left(p, \Pi_{2}^{E} v_{h}\right) \\
& =\sum_{E \in \mathcal{T}_{h}}-f_{E} v_{h} \operatorname{div}(\mathbb{K} \nabla p) \mathrm{d} \boldsymbol{x}+\sum_{E \in \mathcal{T}_{h}} \int_{\partial E} v_{h} \mathbb{K} \nabla p \cdot \boldsymbol{n} \mathrm{d} s \\
& =\sum_{E \in \mathcal{T}_{h}} f_{E} v_{h} f \mathrm{~d} \boldsymbol{x} \\
& =\ell_{h}\left(v_{h}\right)
\end{aligned}
$$

Note that the second term in (41) cancels out as the internal edges of the mesh are visited twice (the normal vector $\boldsymbol{n}$ changes sign each time) and $v_{h}=0$ on the boundary edges. Also, we set $f=-\operatorname{div}(\mathbb{K} \nabla p)$ in the second to last equality. Our numerical studies in fact confirm that the quadratic patch test will be passed up to machine precision errors.

We have provided the details on an explicit construction of the projection map for quadratic elements in the appendix. As for the linear element, it is completely characterized by the two conditions in 38 .

\subsection{Numerical verification}

We proceed to verify that the proposed approach for both linear and quadratic elements does in fact restore optimal convergence. We do this by solving the example problem 20 

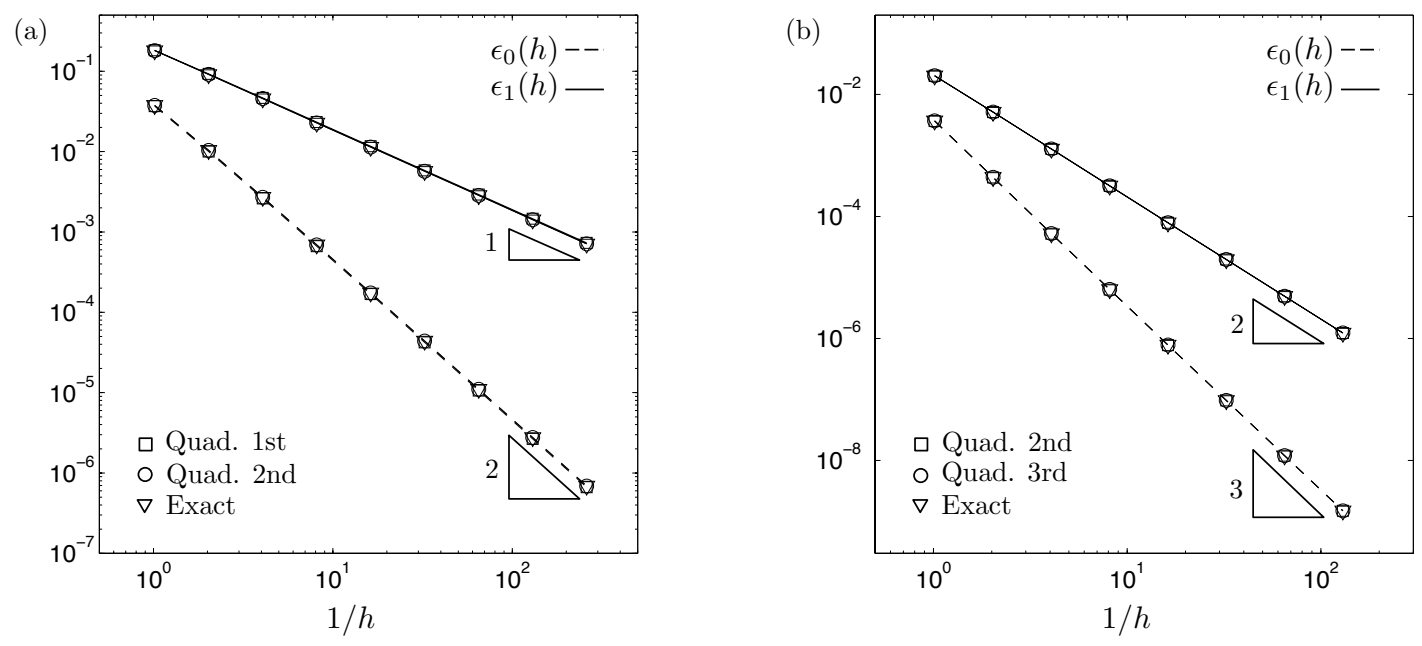

Figure 4: Results of the convergence study with proposed splitting approach with (a) linear elements (b) quadratic elements. In both cases, the quadrangulation scheme with indicated integration order is used

using the proposed discrete bilinear form instead of (11).

As shown in Figure 4(a), we recover optimal convergence rates for linear elements even with the first order quadrature rule. Moreover, the solution errors are nearly identical to those obtained from exact integration, even with the low-order quadrature. In fact, the largest difference in the energy norm errors between the proposed scheme with the first order rule and exact integration is $2.7 \%$. The $L^{2}$-error is slightly smaller with the proposed approach with a difference of $4.0 \%$. This shows that the first order rule, with $n$ integration points for an $n$-gon, can be used in practice without sacrificing accuracy. The use of the more accurate second order error lowers this difference (to $0.027 \%$ and $0.43 \%$ for the energy and $L^{2}$-norm errors, respectively) but requires four times as many integration points.

Figure 4(b) summarize the results for the quadratic elements. The same conclusions can be drawn in this case: optimal convergences rate are restored and the solution errors are almost identical to those with the Galerkin approximation with the exact bilinear. The largest numerical difference between the energy and $L^{2}$-norm errors, with the second order rule, are $0.08 \%$ and $0.80 \%$, respectively.

We remark that the overhead associated with the splitting of the bilinear form is small and the overall cost of construction of $a_{h}^{E}$ is still determined by the number of integration points. For example, observe that (35) requires visiting each edge once, the cost of which is small compared to the geometric construction of gradients of the Wachspress basis functions at each integration point. In our implementation, the overhead associated with splitting of the bilinear form (including the calculation of the projection map) accounts for about $10 \%$ of the total cost of computing the stiffness matrix for both linear and quadratic elements. The major difference between (11) and (29) is that the effort associated with basis function construction and integration is used only on the non-polynomial part of $\mathcal{V}_{m}(E)$ where it is needed. 


\section{Treatment of non-constant coefficients}

We now briefly discuss a possible strategy to handle diffusion tensors with variable coefficients. Such treatment of a position-dependent material function is relevant for a wide range of problems such heat transfer in systems with variable thermal conductivity, flow in porous media with variable permeability, electric conduction with variable resistivity, and magnetostatics with variable magnetic permeability [38, 27, 28. In this general case, we are given a symmetric tensor $\mathbb{K} \in L^{\infty}(\Omega)^{2 \times 2}$ satisfying

$$
\alpha^{-1}|\boldsymbol{\xi}|^{2} \leq \boldsymbol{\xi} \cdot \mathbb{K}(\boldsymbol{x}) \boldsymbol{\xi} \leq \alpha|\boldsymbol{\xi}|^{2}, \quad \forall \boldsymbol{\xi} \in \mathbb{R}^{2}, \forall \boldsymbol{x} \in \Omega
$$

for some positive constant $\alpha$. In order for use of quadrature to make sense, we assume that $\mathbb{K}$ is defined everywhere in the domain.

For each element $E \in \mathcal{T}_{h}$, we construct a first-order approximation to $\mathbb{K}$ over $E$, which we denote by $\mathbb{K}_{E}$. For example, we can do so by setting

$$
\mathbb{K}_{E}=\frac{1}{|E|} \int_{E} \mathbb{K} \mathrm{d} \boldsymbol{x}
$$

or, if $\mathbb{K}$ is a smooth function, we can take $\mathbb{K}_{E}$ to be simply the value of $\mathbb{K}$ the center of $E$. For linear elements, we can proceed as before but with $\mathbb{K}_{E}$ in place of $\mathbb{K}$ without sacrificing first-order convergence rate. For quadratic elements, however, this will lead to loss of an order of convergence. Therefore, we consider the construction of bilinear form that includes a correction term:

$$
\begin{gathered}
a_{h}^{E}(u, v)=\int_{E} \nabla \Pi_{m}^{E} u \cdot \mathbb{K}_{E} \nabla \Pi_{m}^{E} v \mathrm{~d} \boldsymbol{x}+f_{E} \nabla\left(u-\Pi_{m}^{E} u\right) \cdot \mathbb{K}_{E} \nabla\left(v-\Pi_{m}^{E} v\right) \mathrm{d} \boldsymbol{x} \\
+f_{E} \nabla u \cdot\left(\mathbb{K}-\mathbb{K}_{E}\right) \nabla v \mathrm{~d} \boldsymbol{x}
\end{gathered}
$$

Here $\Pi_{m}^{E}$ is defined as in the previous section with $\mathbb{K}$ replaced by $\mathbb{K}_{E}$. With the correction term, we capture the variation of $\mathbb{K}$ inside the element through sampling $\mathbb{K}-\mathbb{K}_{E}$ at the integration points. At the same time, we retain the simplicity offered by a constant tensor in constructing the projection map.

Analysis of the convergence of the resulting approximation can be based on the estimate (23). Here we content ourselves with numerical verification of optimal performance. Borrowing from [13], we consider the problem posed on $\Omega=] 0,1\left[^{2}\right.$ with exact solution

$$
u(\boldsymbol{x})=x_{1}^{3} x_{2}^{2}+x_{1} \sin \left(2 \pi x_{1} x_{2}\right) \sin \left(2 \pi x_{2}\right)
$$

and diffusion tensor given by

$$
\mathbb{K}(\boldsymbol{x})=\left[\begin{array}{cc}
\left(x_{1}+1\right)^{2}+x_{2}^{2} & -x_{1} x_{2} \\
-x_{1} x_{2} & \left(x_{1}+1\right)^{2}
\end{array}\right]
$$

The source function $f$ and boundary data $g$ are prescribed in accordance with (45) and (46). We use the same sequence of regular meshes as before and set $\mathbb{K}_{E}=f_{E} \mathbb{K} \mathrm{d} \boldsymbol{x} /|E|$. The 


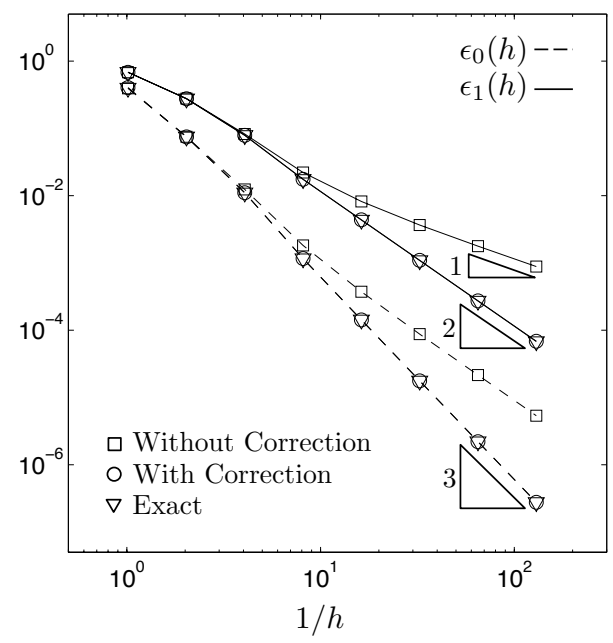

Figure 5: Results of the convergence study for problem $45-46$ using the quadratic elements and second-order quadrangulation scheme

discrete linear form uses the same quadrature that is used for the bilinear form. We omit the results for linear elements because the solution errors, even without the correction term, are very close to the errors obtained with exact integration. Figure (5) summarizes the results for the quadratic elements. It is evident that without the correction term, the rates of convergence are reduced by exactly one order as a result of first-order approximation of $\mathbb{K}$. However, the choice of (44) not only recovers optimal convergence rates, but also leads to nearly the same solution errors as for the exact integration (difference of $<1 \%$ ).

\section{Conclusions}

We conclude by noting that the issue of quadrature error and its adverse effect on convergence is in fact more pronounced in three dimensions. This is because the construction of basis functions for general polyhedral elements is more costly, quadrature rules are more difficult to obtain and the patch test errors are typically larger. The present approach and use of polynomial projections can help overcome the challenges associated with polyhedral finite elements. The proposed approach can also be beneficial for reducing the burden of integration for nonlinear problems where integration of constitutive relations is usually performed at the quadrature points. Finally, the formalism of the polynomial projections is promising in furnishing an alternative way to address the challenges of numerical integration in meshless methods.

\section{Acknowledgements}

This work was inspired by the "Workshop on Discretization Methods for Polygonal and Polyhedral Meshes" which was held in Milan, Italy, from September 17 to 19, 2012. In fact, the inception of this work has its roots in our insightful discussions with Franco 
Brezzi. We also appreciate all the interactions we had with the workshop participants. We acknowledge support from the US National Science Foundation under grant CMMI \#1321661 and from the Donald B. and Elizabeth M. Willett endowment at the University of Illinois at Urbana-Champaign. Any opinion, finding, conclusions or recommendations expressed here are those of the authors and do not necessarily reflect the views of the sponsors.

\section{Appendix A: Construction of polygonal elements}

We discuss the construction of element spaces $\mathcal{V}_{m}(E)$ by means of generalized barycentric coordinates associated with polygon $E$. We will describe the Wachspress coordinates but note that any other set of barycentric coordinates (e.g. Mean Value, harmonic, Sibson, etc.) can be also used. A possibly more economical alternative in the two-dimensional setting makes use of the usual isoparameteric mapping [37]. As discussed in [44], the isoparametric construction in fact defines a new set of barycentric coordinates for polygons. We emphasize that the results of this paper applies to all resulting elements regardless of the choice of the barycentric coordinates.

Suppose $E$ is a strictly convex $n$-gon with vertices located at $\boldsymbol{x}_{1}, \ldots, \boldsymbol{x}_{n}$ oriented counter-clockwise. The Wachspress coordinate associated with the $i$ th vertex is defined in the interior of $E$ by

$$
\varphi_{i}(\boldsymbol{x})=\frac{w_{i}(\boldsymbol{x})}{\sum_{j=1}^{n} w_{j}(\boldsymbol{x})}
$$

with weight functions given by

$$
w_{i}(\boldsymbol{x})=\frac{A\left(\boldsymbol{x}_{i-1}, \boldsymbol{x}_{i}, \boldsymbol{x}_{i+1}\right)}{A\left(\boldsymbol{x}_{i-1}, \boldsymbol{x}_{i}, \boldsymbol{x}\right) A\left(\boldsymbol{x}_{i}, \boldsymbol{x}_{i+1}, \boldsymbol{x}\right)}
$$

Here $A(\boldsymbol{a}, \boldsymbol{b}, \boldsymbol{c})$ denotes the area of the triangle with vertices located at points $\boldsymbol{a}, \boldsymbol{b}$ and $\boldsymbol{c}$. We are using the convention that $\boldsymbol{x}_{n+1}=\boldsymbol{x}_{1}$ and $\boldsymbol{x}_{0}=\boldsymbol{x}_{n}$. It is evident that $\varphi_{i}$ 's are positive functions that form a partition of unity in $E^{\circ}$. Moreover, one can show

$$
\sum_{i=1}^{n} \boldsymbol{x}_{i} \varphi_{i}(\boldsymbol{x})=\boldsymbol{x}, \quad \forall \boldsymbol{x} \in E^{\circ}
$$

From these properties, it follows that Wachspress functions can be extended continuously to $\partial E$ such that 18

$$
\varphi_{i}(\boldsymbol{x})=1-\frac{\left|\boldsymbol{x}-\boldsymbol{x}_{i}\right|}{\left|\boldsymbol{x}_{i+1}-\boldsymbol{x}_{i}\right|}, \quad \varphi_{i+1}(\boldsymbol{x})=\frac{\left|\boldsymbol{x}-\boldsymbol{x}_{i}\right|}{\left|\boldsymbol{x}_{i+1}-\boldsymbol{x}_{i}\right|}, \quad \varphi_{j}(\boldsymbol{x})=0, \forall j \neq i, i+1
$$

if $\boldsymbol{x}$ lies on the edge connecting $\boldsymbol{x}_{i}$ and $\boldsymbol{x}_{i+1}$. Note that (50) implies that Wachspress coordinates satisfy the Kronecker-delta property, i.e., $\varphi_{i}\left(\boldsymbol{x}_{j}\right)=\delta_{i j}$ and vary linearly on 
$\partial E 7$. Subsequently, we have the linear precision property of

$$
p(\boldsymbol{x})=\sum_{i=1}^{n} p\left(\boldsymbol{x}_{i}\right) \varphi_{i}(\boldsymbol{x}), \quad \forall p \in \mathcal{P}_{1}(E)
$$

for any point $\boldsymbol{x}$ in the closure of $E$. We set the linear element space for $E$ as $\mathcal{V}_{1}(E)=$ $\operatorname{span}\left\{\varphi_{1}, \ldots, \varphi_{n}\right\}$. Observe how (51) implies (6) with $m=1$.

To construct the quadartic serendipity element on $E$, we first define mid-side nodes $\hat{\boldsymbol{x}}_{i}=\left(\boldsymbol{x}_{i}+\boldsymbol{x}_{i+1}\right) / 2$. The basis functions for $\mathcal{V}_{2}(E)$ are given by

$$
\psi_{i}(\boldsymbol{x})=\sum_{a=1}^{n} \sum_{b=1}^{n} c_{i}^{a b} \varphi_{a}(\boldsymbol{x}) \varphi_{b}(\boldsymbol{x}), \quad i=1, \ldots, 2 n
$$

where $\varphi_{a}$ are barycentric coordinates for $E$ and coefficients $c_{i}^{a b}$ are chosen such that

$$
p(\boldsymbol{x})=\sum_{i=1}^{n}\left[p\left(\boldsymbol{x}_{i}\right) \psi_{i}(\boldsymbol{x})+p\left(\hat{\boldsymbol{x}}_{i}\right) \psi_{i+n}(\boldsymbol{x})\right], \quad \forall p \in \mathcal{P}_{2}(E)
$$

and Kronecker-delta property is satisfied 8 . In [31, it is shown that a stable choice of coefficients $c_{i}^{a b}$ exists and a procedure for computing them is presented. As a result of this construction, the basis functions exhibit quadratic variation on the boundary and (6) is satisfied for $m=2$.

\section{Appendix B: Implementation aspects}

We will provide details on the algebraic construction of the projection map and the discrete bilinear forms for both linear and quadratic elements. The presentation proceeds along similar lines as [35] where implementation of a first order VEM formulation for Poisson's problem is discussed.

We unify the presentation by noting that the right hand side of $(38)$ reduces to $\int_{\partial E} v \mathbb{K} \nabla p$. $\boldsymbol{n} \mathrm{d} s=a^{E}(v, p)$ whenever $p \in \mathcal{P}_{1}(E)$. Therefore, the first condition in the definition of the projection maps is taken to $b e^{9}$

$$
a^{E}\left(p, \Pi_{m}^{E} v\right)=-f_{E} v \operatorname{div}(\mathbb{K} \nabla p) \mathrm{d} \boldsymbol{x}+\int_{\partial E} v \mathbb{K} \nabla p \cdot \boldsymbol{n} \mathrm{d} s, \quad \forall p \in \mathcal{P}_{m}(E)
$$

for both linear and quadratic elements. We can also replace the second condition in 26 with an equivalent condition given by:

$$
\overline{\Pi_{m}^{E} v}=\bar{v}, \quad \forall v \in \mathcal{V}_{m}(E)
$$

\footnotetext{
${ }^{7}$ These properties are essential ingredients in constructing the conforming finite element space (5) with degrees of freedom associated with the vertices of the mesh.

${ }^{8}$ That is, $\psi_{i}\left(\boldsymbol{x}_{j}\right)=\psi_{i+n}\left(\hat{\boldsymbol{x}}_{j}\right)=\delta_{i j}$ and $\psi_{i}\left(\hat{\boldsymbol{x}}_{j}\right)=\psi_{i+n}\left(\boldsymbol{x}_{j}\right)=0$

${ }^{9}$ For the linear element, this term vanishes. For the quadratic element, as in section 5.2, we assume that the quadrature used for the first term is exact when $v \in \mathcal{P}_{2}(E)$.
} 
The equivalence follows from the fact that (54) implies that $\nabla \Pi_{m}^{E} p=\nabla p$ for $p \in \mathcal{P}_{m}(E)$. Thus, (54) together with (55) ensures that the projection fixes polynomial functions.

Let $n_{v}=\operatorname{dim} \mathcal{V}_{m}(E)$ and $n_{p}=\operatorname{dim} \mathcal{P}_{m}(E)-1$, and consider a basis for $\mathcal{P}_{m}(E)$ of the form $\left\{1, p_{1}, \ldots, p_{n_{p}}\right\}$ such that $\overline{p_{\alpha}}=0$ for $\alpha=1, \ldots, n_{p}$. For example,

$$
p_{0}(\boldsymbol{x})=1, \quad p_{1}(\boldsymbol{x})=x_{1}-\overline{x_{1}}, \quad p_{2}(\boldsymbol{x})=x_{2}-\overline{x_{2}}
$$

is such a basis for $\mathcal{P}_{1}(E)$. As before, let $\left\{\varphi_{1}, \ldots, \varphi_{n_{v}}\right\}$ be the canonical basis for $\mathcal{V}_{m}(E)$.

We define two matrices $\boldsymbol{R}$ and $\boldsymbol{N}$ of size $n_{v} \times n_{p}$ by

$$
\begin{aligned}
\boldsymbol{R}_{i \alpha} & =-f_{E} \varphi_{i} \operatorname{div}\left(\mathbb{K} \nabla p_{\alpha}\right) \mathrm{d} \boldsymbol{x}+\int_{\partial E} \varphi_{i} \mathbb{K} \nabla p_{\alpha} \cdot \boldsymbol{n} \mathrm{d} s \\
\boldsymbol{N}_{i \alpha} & =p_{\alpha}\left(\boldsymbol{x}_{i}\right)
\end{aligned}
$$

where $\boldsymbol{x}_{i}$ is the location of the $i$ th node of $E$ (associated with $\varphi_{i}$ ). Observe that $\boldsymbol{R}_{i \alpha}$ is the right-hand-side of (54) for $v=\varphi_{i}$ and $p=p_{\alpha}$. Also the Lagrangian property of the basis functions and their polynomial precision implies

$$
p_{\alpha}(\boldsymbol{x})=\sum_{i=1}^{n_{v}} \boldsymbol{N}_{i \alpha} \varphi_{i}(\boldsymbol{x})
$$

Using (57)- (59) and the exactness of the quadrature rule on polynomials, we have

$$
\begin{aligned}
a^{E}\left(p_{\alpha}, p_{\beta}\right) & =-\int_{E} p_{\alpha} \operatorname{div}\left(\mathbb{K} \nabla p_{\beta}\right) \mathrm{d} \boldsymbol{x}+\int_{\partial E} p_{\alpha} \mathbb{K} \nabla p_{\beta} \cdot \boldsymbol{n} \mathrm{d} s \\
& =-f_{E} p_{\alpha} \operatorname{div}\left(\mathbb{K} \nabla p_{\beta}\right) \mathrm{d} \boldsymbol{x}+\int_{\partial E} p_{\alpha} \mathbb{K} \nabla p_{\beta} \cdot \boldsymbol{n} \mathrm{d} s \\
& =\sum_{i=1}^{n_{v}} \boldsymbol{N}_{i \alpha}\left[-f_{E} \varphi_{i} \operatorname{div}\left(\mathbb{K} \nabla p_{\beta}\right) \mathrm{d} \boldsymbol{x}+\int_{\partial E} \varphi_{i} \mathbb{K} \nabla p_{\beta} \cdot \boldsymbol{n} \mathrm{d} s\right] \\
& =\left[\boldsymbol{N}^{T} \boldsymbol{R}\right]_{\alpha \beta}
\end{aligned}
$$

This shows that $\boldsymbol{N}^{T} \boldsymbol{R}$ is an $n_{p} \times n_{p}$ symmetric positive definite matrix.

Since $\Pi_{m}^{E} \varphi_{i}$ is an element of $\mathcal{P}_{m}(E)$, there exists an $n_{v} \times n_{p}$ matrix $\boldsymbol{S}$ such that:

$$
\Pi_{m}^{E} \varphi_{i}=\frac{1}{n_{v}}+\sum_{\beta=1}^{n_{p}} \boldsymbol{S}_{i \beta} p_{\beta}
$$

for $i=1, \ldots, n_{v}$. Note that $\overline{\Pi_{m}^{E} \varphi_{i}}=1 / n_{v}=\overline{\varphi_{i}}$ and so 55 is satisfied. To derive an expression for $\boldsymbol{S}$, we appeal to (54), and set $p=p_{\alpha}$ and $v=\varphi_{i}$ to get

$$
\sum_{\beta=1}^{n_{p}} \boldsymbol{S}_{i \beta} a^{E}\left(p_{\alpha}, p_{\beta}\right)=\boldsymbol{R}_{i \alpha}
$$

Here we have used the linearity of the bilinear form and expansion (61). 
From $(60)$ and the fact that 620 must hold for $\alpha=1, \ldots, n_{p}$, we obtain following expression for $\boldsymbol{S}$

$$
\boldsymbol{S}=\boldsymbol{R}\left(\boldsymbol{N}^{T} \boldsymbol{R}\right)^{-1}
$$

which in turn, through (61), gives the expression for the projection map. One can verify that for $m=1$, 61) and (63) recover the expression (35) derived in section 5.1.

We proceed to derive explicit algebraic expressions for the stiffness matrix associated with bilinear $a_{h}^{E}$. Recall that the $(i, j)$ th entry of the stiffness matrix associated with $E$ is given by

$$
a_{h}^{E}\left(\varphi_{i}, \varphi_{j}\right)=a^{E}\left(\Pi_{m}^{E} \varphi_{i}, \Pi_{m}^{E} \varphi_{j}\right)+f_{E} \nabla\left(\varphi_{i}-\Pi_{m}^{E} \varphi_{i}\right) \cdot \mathbb{K} \nabla\left(\varphi_{j}-\Pi_{m}^{E} \varphi_{j}\right) \mathrm{d} \boldsymbol{x}
$$

We can compute the first term of the stiffness matrix as follows

$$
a^{E}\left(\Pi_{m}^{E} \varphi_{i}, \Pi_{m}^{E} \varphi_{j}\right)=\sum_{\alpha=1}^{n_{p}} \sum_{\beta=1}^{n_{p}} \boldsymbol{S}_{i \alpha} \boldsymbol{S}_{j \beta} a^{E}\left(p_{\alpha}, p_{\beta}\right)=\left[\boldsymbol{S}\left(\boldsymbol{N}^{T} \boldsymbol{R}\right) \boldsymbol{S}^{T}\right]_{i j}=\left[\boldsymbol{R}\left(\boldsymbol{N}^{T} \boldsymbol{R}\right)^{-1} \boldsymbol{R}^{T}\right]_{i j}
$$

To get an expression for the second term, we note that

$$
\begin{aligned}
\varphi_{i}-\Pi_{m}^{E} \varphi_{i} & =\varphi_{i}-\frac{1}{n_{v}}-\sum_{\beta=1}^{n_{p}} \boldsymbol{S}_{i \beta} \sum_{j=1}^{n_{v}} \boldsymbol{N}_{j \beta} \varphi_{j} \\
& =\varphi_{i}-\frac{1}{n_{v}}-\sum_{j=1}^{n_{v}}\left(\boldsymbol{S} \boldsymbol{N}^{T}\right)_{i j} \varphi_{j} \\
& =\sum_{j=1}^{n_{v}}\left(\boldsymbol{I}_{i j}-\frac{1}{n_{v}} \boldsymbol{U}_{i j}-\left[\boldsymbol{R}\left(\boldsymbol{N}^{T} \boldsymbol{R}\right)^{-1} \boldsymbol{N}^{T}\right]_{i j}\right) \varphi_{j}
\end{aligned}
$$

where $\boldsymbol{I}$ denotes the $n_{v} \times n_{v}$ identity matrix and $\boldsymbol{U}$ is the $n_{v} \times n_{v}$ matrix with unit entries. Defining,

$$
\boldsymbol{P}:=\boldsymbol{I}-\frac{1}{n_{v}} \boldsymbol{U}-\boldsymbol{R}\left(\boldsymbol{N}^{T} \boldsymbol{R}\right)^{-1} \boldsymbol{N}^{T}
$$

we have $\varphi_{i}-\Pi_{m}^{E} \varphi_{i}=\sum_{j=1}^{n_{v}} \boldsymbol{P}_{i j} \varphi_{j}$. In turn, the second term of 64 can be written as

$$
f_{E} \nabla\left(\varphi_{i}-\Pi_{m}^{E} \varphi_{i}\right) \cdot \mathbb{K} \nabla\left(\varphi_{j}-\Pi_{m}^{E} \varphi_{j}\right) \mathrm{d} \boldsymbol{x}=\sum_{k=1}^{n_{v}} \sum_{\ell=1}^{n_{v}} \boldsymbol{P}_{i k} \boldsymbol{P}_{j \ell}\left(f_{E} \nabla \varphi_{k} \cdot \mathbb{K} \nabla \varphi_{\ell} \mathrm{d} \boldsymbol{x}\right)
$$

Observe that the term in the parenthesis is $(k, \ell)$ th entry of the usual stiffness matrix obtained from quadrature (i.e., the stiffness matrix corresponding to bilinear form (11)). Defining $\boldsymbol{K}_{k \ell}:=f_{E} \nabla \varphi_{k} \cdot \mathbb{K} \nabla \varphi_{\ell} \mathrm{d} \boldsymbol{x}$, the expression for the stiffness matrix reduces to

$$
a_{h}^{E}\left(\varphi_{i}, \varphi_{j}\right)=\left[\boldsymbol{R}\left(\boldsymbol{N}^{T} \boldsymbol{R}\right)^{-1} \boldsymbol{R}^{T}+\boldsymbol{P} \boldsymbol{K} \boldsymbol{P}^{T}\right]_{i j}
$$

For case of non-constant coefficients, the matrix $\boldsymbol{R}$ is defined as (57) but with $\mathbb{K}$ replaced by $\mathbb{K}_{E}$. Setting $\tilde{\boldsymbol{K}}_{i j}=f_{E} \nabla \varphi_{i} \cdot \mathbb{K} \nabla \varphi_{j} \mathrm{~d} \boldsymbol{x}$ and $\boldsymbol{K}_{i j}=f_{E} \nabla \varphi_{i} \cdot \mathbb{K}_{E} \nabla \varphi_{j} \mathrm{~d} \boldsymbol{x}$, the stiffness matrix associated with the corrected bilinear (44) is

$$
a_{h}^{E}\left(\varphi_{i}, \varphi_{j}\right)=\left[\boldsymbol{R}\left(\boldsymbol{N}^{T} \boldsymbol{R}\right)^{-1} \boldsymbol{R}^{T}+\boldsymbol{P} \boldsymbol{K} \boldsymbol{P}^{T}+(\tilde{\boldsymbol{K}}-\boldsymbol{K})\right]_{i j}
$$


where $\boldsymbol{P}$ is again defined by $(67)$.

\section{References}

\section{References}

[1] B. Ahmad, A. Alsaedi, F. Brezzi, L. D. Marini, and A. Russo, Equivalent projectors for Virtual Element Methods, Comput Math Appl, To appear (2013).

[2] I. BabušKa, U. BanerJee, J. E. Osborn, And Q. Li, Quadrature for meshless methods, Int. J. Numer. Meth. Engng, 76 (2008), pp. 1434-1470.

[3] I. Babuška, U. Banerjee, J. E. Osborn, and Q. Zhang, Effect of numerical integration on meshless methods, Computer Methods in Applied Mechanics and Engineering, 198 (2009), pp. 2886-2897.

[4] F. Bassi, L. Botti, A. Colombo, D. A. Di Pietro, and P. Tesini, On the flexibility of agglomeration based physical space discontinuous Galerkin discretizations, Journal of Computational Physics, 231 (2012), pp. 45-65.

[5] L. Beirão Da Veiga, F. Brezzi, A. Cangiani, G. Manzini, L. D. Marini, AND A. Russo, Basic principles of Virtual Element Methods, Mathematical Models and Methods in Applied Sciences, 23 (2013), pp. 199-214.

[6] L. Beirão Da Veiga and K. Lipnikov, A mimetic discretization of the Stokes problem with selected edge bubbles, SIAM J Sci Comput, 32 (2010), pp. 875-893.

[7] L. Beirão Da Veiga, K. Lipnikov, and G. Manzini, Arbitrary-order nodal mimetic discretizations of elliptic problems on polygonal meshes, SIAM J Numer Anal, 49 (2011), pp. 1737-1760.

[8] L. Beirão da Veiga and G. Manzini, An a posteriori error estimator for the mimetic finite difference approximation of elliptic problems, Int J Numer Meth Engng, 76 (2008), pp. 1696-1723.

[9] J. E. Bishop, Simulating the pervasive fracture of materials and structures using randomly close packed voronoi tessellations, Comput Mech, 44 (2009), pp. 455-471.

[10] L. V. Branets, S. S. Ghai, S. L. Lyons, And X.-H. Wu, Challenges and technologies in reservoir modeling, Communications in Computational Physics, 6 (2009), pp. 1-23.

[11] S. C. Brenner and L. R. Scott, The Mathematical Theory of Finite Element Methods, Springer, 2nd ed., 2002.

[12] F. Brezzi, A. Buffa, And K. Lipnikov, Mimetic finite differences for elliptic problems, ESAIM Math. Model. Numer. Anal., 43 (2009), pp. 277-295. 
[13] F. Brezzi, K. Lipnikov, and V. Simoncini, A family of mimetic finite difference methods on polygonal and polyhedral meshes, Mathematical Models and Methods in Applied Sciences, 15 (2005), pp. 1533-1551.

[14] J.-S. Chen, C.-T. Wu, S. Yoon, And Y. You, A stabilized conforming nodal integration for galerkin mesh-free methods, International Journal for Numerical Methods in Engineering, 50 (2001), pp. 435-466.

[15] P. G. Ciarlet, Finite Element Method for Elliptic Problems, Society for Industrial and Applied Mathematics, Philadelphia, PA, USA, 2002.

[16] L. Codechasa, R. Specogna, And F. Trevisan, A new set of basis functions for the discrete geometric approach, Journal of Computational Physics, 229 (2010), pp. 7401-7410.

[17] M. S. Ebeida And S. A. Mitchell, Uniform random Voronoi meshes, in Proceedings of the 20th International Meshing Roundtable, 2012, pp. 273-290.

[18] M. Floater, K. Hormann, And G. Kos, A general construction of barycentric coordinates over convex polygons, Adv Comput Math, 24 (2006), pp. 311-331.

[19] A. Gillette And C. BAJAJ, Dual formulations of mixed finite element methods with applications, Computer-Aided Design, 43 (2011), pp. 1213-1221.

[20] A. Gillette, A. Rand, And C. Bajaj, Error estimates for generalized barycentric interpolation, Adv Comput Math, 37 (2012), pp. 417-439.

[21] A. N. Hirani, K. B. Nakshatrala, and J. H. Chaudhry, Numerical method for Darcy flow derived using Discrete Exterior Calculus, arXiv preprint arXiv:0810.3434, (2008).

[22] B. Jerome And E. Alexandre, Analysis of compatible discrete operator schemes for elliptic problems on polyhedral meshes, arXiv preprint arXiv:1211.3354, (2012).

[23] S. E. Leon, D. W. Spring, and G. H. Paulino, Reduction in mesh bias for dynamic fracture using adaptive splitting of polygonal finite elements, Int J Numer Meth Engng, (submitted).

[24] Y. Liu And T. BelytschKo, A new support integration scheme for the weakform in mesh-free methods, Int J Numer Meth Engng, 82 (2010), pp. 699-715.

[25] S. E. Mousavi, H. XiaO, And N. Sukumar, Generalized Gaussian quadrature rules on arbitrary polygons, Int J Numer Meth Eng, 82 (2010), pp. 99-113.

[26] S. Natarajan, S. Bordas, And D. R. MAhapatra, Numerical integration over arbitrary polygonal domains based on Schwarz-Christoffel conformal mapping, Int J Numer Meth Eng, 80 (2009), pp. 103-134.

[27] G. H. Paulino, Fracture of functionally graded materials, Eng Fract Mech, Special Issue on Functionally Graded Materials, 69 (2002). 
[28] — Modeling of functionally graded materials, Int J Comput Eng Sci, Special Issue, 5 (2004).

[29] G. H. Paulino, K. Park, W. Celes, and R. Espinha, Adaptive dynamic cohesive fracture simulation using nodal perturbation and edge-swap operators, Int J Numer Meth Engng, 84 (2010), pp. 1303-1343.

[30] M. Puso, J. Chen, E. Zywicz, And W. Elmer, Meshfree and finite element nodal integration methods, International Journal for Numerical Methods in Engineering, 74 (2008), pp. 416-446.

[31] A. Rand, A. Gillette, And C. BajaJ, Quadratic serendipity finite elements on polygons using generalized barycentric coordinates, arXiv preprint arXiv:1109.3259, (2011).

[32] M. M. Rashid And M. Selimotic, A three-dimensional finite element method with arbitrary polyhedral elements, Int. J. Numer. Meth. Engng, 67 (2006), pp. 226-252.

[33] J. J. Rimoli, J. J. Rojas, and F. N. Khemani, On the mesh dependency of cohesive zone models for crack propagation analysis, in Structural Dynamics and Materials Conference, 2012.

[34] S. RJASANOW AND S. WEIBER, Higher order BEM-based FEM on polygonal meshes, SIAM Journal on Numerical Analysis, 50 (2012), pp. 2357-2378.

[35] A. Russo, Virtual Element Methods II, in Conference on Discretization Methods for Polygonal and Polyhedral Meshes, Milan, Italy, 2012.

[36] N. Sukumar And E. A. MaLsch, Recent advances in the construction of polygonal finite element interpolants, Arch Comput Method E, 13 (2006), pp. 129-163.

[37] N. Sukumar and A. Tabarraei, Conforming polygonal finite elements, Int J Numer Meth Eng, 61 (2004), pp. 2045-2066.

[38] A. Sutradhar and G. H. Paulino, A simple boundary element method for problems of potential in non-homogeneous media, Int J Numer Meth Eng, 60 (2004), pp. 2203-2230.

[39] A. Tabarraei and N. Sukumar, Adaptive computations on conforming quadtree meshes, Finite Elements in Analysis and Design, 41 (2005), pp. 686-702.

[40] C. Talischi, G. H. Paulino, and C. H. Le, Honeycomb Wachspress finite elements for structural topology optimization, Struct Multidisc Optim, 37 (2009), pp. 569-583.

[41] C. Talischi, G. H. Paulino, A. Pereira, and I. F. M. Menezes, Polygonal finite elements for topology optimization: A unifying paradigm, Int J Numer Meth Eng, 82 (2010), pp. 671-698.

[42] —_ PolyMesher: A general-purpose mesh generator for polygonal elements written in Matlab, Struct Multidisc Optim, 45 (2012), pp. 309-328. 
[43] _ PolyTop: a Matlab implementation of a general topology optimization framework using unstructured polygonal finite element meshes, Struct Multidisc Optim, 45 (2012), pp. 329-357.

[44] C. Talischi, A. Pereira, G. H. Paulino, I. F. M. Menezes, and M. S. CarVALHO, Polygonal finite elements for incompressible flow, Int J Numer Meth Fl, (accepted).

[45] E. L. Wachspress, A Rational Finite Element Basis, Academic Press, 1975.

[46] Q. Zhang And U. BanerJee, Numerical integration in Galerkin meshless methods, applied to elliptic Neumann problem with non-constant coefficients, Advances in Computational Mathematics, 37 (2012), pp. 453-492. 